\title{
Micro-CT Mineral Density Profile as Reference Standard for Early Carious Lesion Activity Assessment.
}

\section{Adam Michael Hoxie}

Division of Diagnostic Sciences, Adams School of Dentistry, University of North Carolina at Chapel Hill Aline de Almeida Neves

Department of Pediatric Dentistry and Orthodontics, Federal University of Rio de Janeiro

\section{Kevin Moss}

Division of Oral and Craniofacial Health Sciences, Adams School of Dentistry, University of North Carolina at Chapel Hill

\section{Adalberto Bastos de Vasconcellos}

Division of Comprehensive Oral Health, Operative Dentistry, Dentistry, Adams School of Dentistry, University of North Carolina at Chapel Hill

\section{Andrea Ferreira-Zandona}

Department of Comprehensive Care, Tufts University School of Dental Medicine

Apoena Aguiar Ribeiro ( $\sim$ apoena@email.unc.edu )

Division of Diagnostic Sciences, Adams School of Dentistry, University of North Carolina at Chapel Hill

\section{Research Article}

Keywords: clinical caries diagnosis, treatment decisions, Micro-CT Mineral Density, ICDAS 1-3, micro-CT

Posted Date: June 1st, 2021

DOl: https://doi.org/10.21203/rs.3.rs-558240/v1

License: (c) (1) This work is licensed under a Creative Commons Attribution 4.0 International License. Read Full License 


\title{
Micro-CT Mineral Density Profile as Reference Standard for Early Carious Lesion Activity Assessment
}

\author{
Adam Michael Hoxie ${ }^{a}$, Aline Almeida Neves ${ }^{b}$, Kevin Moss ${ }^{c}$, Adalberto Bastos de \\ Vasconcellos $^{\mathrm{d}}$, Andrea Ferreira Zandona ${ }^{\mathrm{e}}$, Apoena Aguiar Ribeiro ${ }^{\mathrm{a}^{*}}$

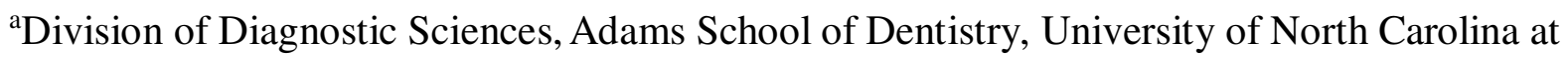 \\ Chapel Hill, Chapel Hill, NC 27599, USA. \\ bepartment of Pediatric Dentistry and Orthodontics, Federal University of Rio de Janeiro, \\ Brazil. Centre for Oral Clinical and Translational Sciences, Faculty of Dentistry, Oral \& \\ Craniofacial Sciences, King's College London, Denmark Hill, London, SE5 9RS, England. \\ 'Statistics, Division of Oral and Craniofacial Health Sciences, Adams School of Dentistry, \\ University of North Carolina at Chapel Hill, Chapel Hill, NC 27599, USA. \\ 'Division of Comprehensive Oral Health, Operative Dentistry, Adams School of Dentistry, \\ University of North Carolina at Chapel Hill, Chapel Hill, NC 27599, USA. \\ e Department of Comprehensive Care, Tufts University School of Dental Medicine, Boston, MA \\ 02111, USA. \\ *apoena@email.unc.edu
}




\section{ABSTRACT}

A challenging and crucial component to clinical caries diagnosis is diagnosing the activity of early lesions because it may have a significant impact on treatment decisions. This study aims to utilize microtomographic (micro-CT) scans of the enamel smooth surface layer to serve as a reference standard for future ex vivo caries activity assessment validation studies. Sound and noncavitated (ICDAS 1-3) smooth surfaces $(n=59)$ of extracted permanent teeth were examined for caries activity by calibrated individuals via visual-tactile examination. Each surface was scanned via micro-CT and line plot analysis generated plots of the mineral density against lesion depth, where the AUC was calculated. AUC thresholds were established to classify sound, remineralized, and demineralized surfaces against the gold standard examiner's ICCMS assessment of sound, inactive, and active lesions, respectively. Diagnostic thresholds were associated with caries ICCMS activity classification using AUC mineral density values of the most external $96 \mu \mathrm{m}$ of enamel. The established thresholds demonstrated $76.3 \%$ agreement with the ICCMS assessment in identifying demineralized lesions $(\mathrm{k}=0.45)$, with high sensitivity (0.73) and specificity (0.77). This study demonstrates quantifiable differences among demineralized, remineralized lesions, and sound surfaces, which contributes to the establishment of micro-CT as a reference standard for caries activity that may be used to improve clinical and technological caries examinations.

\section{Introduction}

Detection of initial carious lesions has become increasingly important in modern dentistry as it supports effective remineralization therapies, prevents loss of sound tooth structure and maintains the tooth's lifespan. A crucial component in diagnosing early lesions is to determine caries activity, as active lesions can be arrested by non-invasive treatments, preventing further 
progression. Detection of early caries activity currently relies on visual-tactile methods, such as the International Caries Classification and Management System (ICCMS), which includes careful visual examination with loupes and tactile examination with a WHO probe to assess lesion activity based on surface texture, location relative to plaque stagnation area, and color [1]. However, discrepancies exist between visual-tactile methods such as ICCMS, Nyvad et al. [2], and Ekstrand et al. [3] criteria. Additionally, while these systems can adequately assess the severity of initial lesions with relatively good consistency, intra- and inter-rater reliability is significantly lower for them when used to assess lesion activity [4]. Therefore, there is a need to improve reliability of caries activity detection methods, especially for initial non-cavitated carious lesions, in which the activity diagnosis will affect the treatment options [5].

The only definite indication of an active lesion is further demineralization in vivo, a gold standard that is difficult to replicate in controlled studies. Thus, a need remains for an ex vivo reference standard for initial lesion demineralization that could be applied to calibration sessions for examiners involved in scoring caries activity, as well as well-controlled studies that will someday improve reliability of clinical activity assessment methods. One method currently employed as a reference standard is the application of aqueous methyl red to a histologically sectioned lesion. This method relies on the higher acid content, and lower $\mathrm{pH}$, of active lesions as an ex vivo marker of caries activity [6,7]. However, this method leads to the permanent destruction of samples for analysis and has failed to gain full acceptance in the literature as a well-established standard [8].

One novel approach with potential use as a caries activity reference standard for smooth surfaces is microcomputed tomography (micro-CT) [9]. Advantages of micro-CT include its nondestructive nature (allowing sample preservation), production of high-resolution images in three 
dimensions, and ability to analyze the internal structure of lesions from any desired location. While micro-CT analysis has been well studied for various applications, such as determining lesion severity and tooth anatomy [10-12], only preliminary work has been completed regarding its utility for assessing caries activity [13-15]. Differences in mineral content of the enamel surface layer on micro-CT have been observed between active and arrested early carious lesions, with active lesions demonstrating a characteristic demineralization pattern [15,16]. This finding corresponds to the surface layer hypothesis for remineralization proposed by Groenveld and Arends $[17,18]$. Thus, this study aims to follow-up on previous qualitative findings from the literature, using a significantly larger sample size, by comparing demineralization and remineralization patterns of active and arrested (or inactive) initial enamel lesions. Specifically, this study aims to identify quantitative differences between mineral density profiles on smooth surfaces clinically classified as non-cavitated active lesions, inactive lesions, and sound surfaces, in an effort to identify a proper reference standard for future caries activity standardized studies.

\section{Methods}

\section{Sample Selection}

Extracted human teeth with non-cavitated (ICDAS 1-3) smooth coronal surfaces $(\mathrm{n}=59)$ were selected based on visual assessment. The selected teeth were unrestored and free of developmental defects. They were stored in $0.2 \%$ thymol solution at $4{ }^{\circ} \mathrm{C}$. This study was approved by the institutional University of North Carolina- Chapel Hill Office of Human Research Ethics (study \#18-1654) and was classified as NHSR (designated as exempt given that it does not constitute human subjects research). 


\section{Sample Preparation}

The selected teeth were cleaned via hand scaling, further polished with Robinson brush and deionized water (DIW), rinsed and stored in DIW. This was changed every 3 days for 3 cycles, while still being stored at $4^{\circ} \mathrm{C}$. After taking a white light image of each tooth (Nikon D5200, AFS Micro Nikkor $85 \mathrm{~mm}$ lens, f/32, Melville, NY), a slide was created for each tooth that included a masked area indicating the surface area which examiners were asked to evaluate.

\section{Examiner Calibration}

Two experienced dentists reviewed the online ICDAS Program (ICDAS Calibration for ICCMS(TM) - https://www.iccms-web.com/course) and discussed the activity criteria in a training session using a set of practice teeth with active and inactive lesions. Then, they independently assessed a set of 13 smooth surfaces on practice teeth using ICCMS to assess inter-examiner agreement for calibration. Each examiner repeated the full exam at least one week after initial completion to assess intra-examiner agreement. The intra-examiner kappa coefficient (k) ranged from 0.64-0.76 with inter-examiner $k=0.76$.

\section{ICCMS Examination}

The two examiners independently used magnification (x2.5), a headlight, air syringe for 5 seconds, and a WHO probe for the visual-tactile assessment. Each examiner evaluated the teeth ( $n=59)$ twice, with a week interval, and graded the surface of interest as active, inactive or sound, using ICCMS activity criteria: active lesions showed white to yellow color with opaque appearance, loss of luster and rough texture; inactive lesions showed white, brown or black color, with shiny appearance, and smooth texture [19]. 


\section{Micro-CT Scanning}

The teeth ( $\mathrm{n}=59$ ) were scanned using a high-energy micro-CT (Scanco $\mu \mathrm{CT} 40$, Scanco Medical, Switzerland). The acquisition parameters were defined as: $70 \mathrm{kV}, 114 \mu \mathrm{A}$, isotropic voxel size $16 \mu \mathrm{m}$, scanned in air, with 250 projections per slice, and an integration time of $200 \mathrm{~ms}$. After acquisition, the data were reconstructed with Scanco software (Scanco Medical, Switzerland) using a back projection algorithm. Figure 1d-f includes image slices that have been oriented to contain the deepest part of the lesion, corresponding to the clinical pictures in Figure 1a-c.

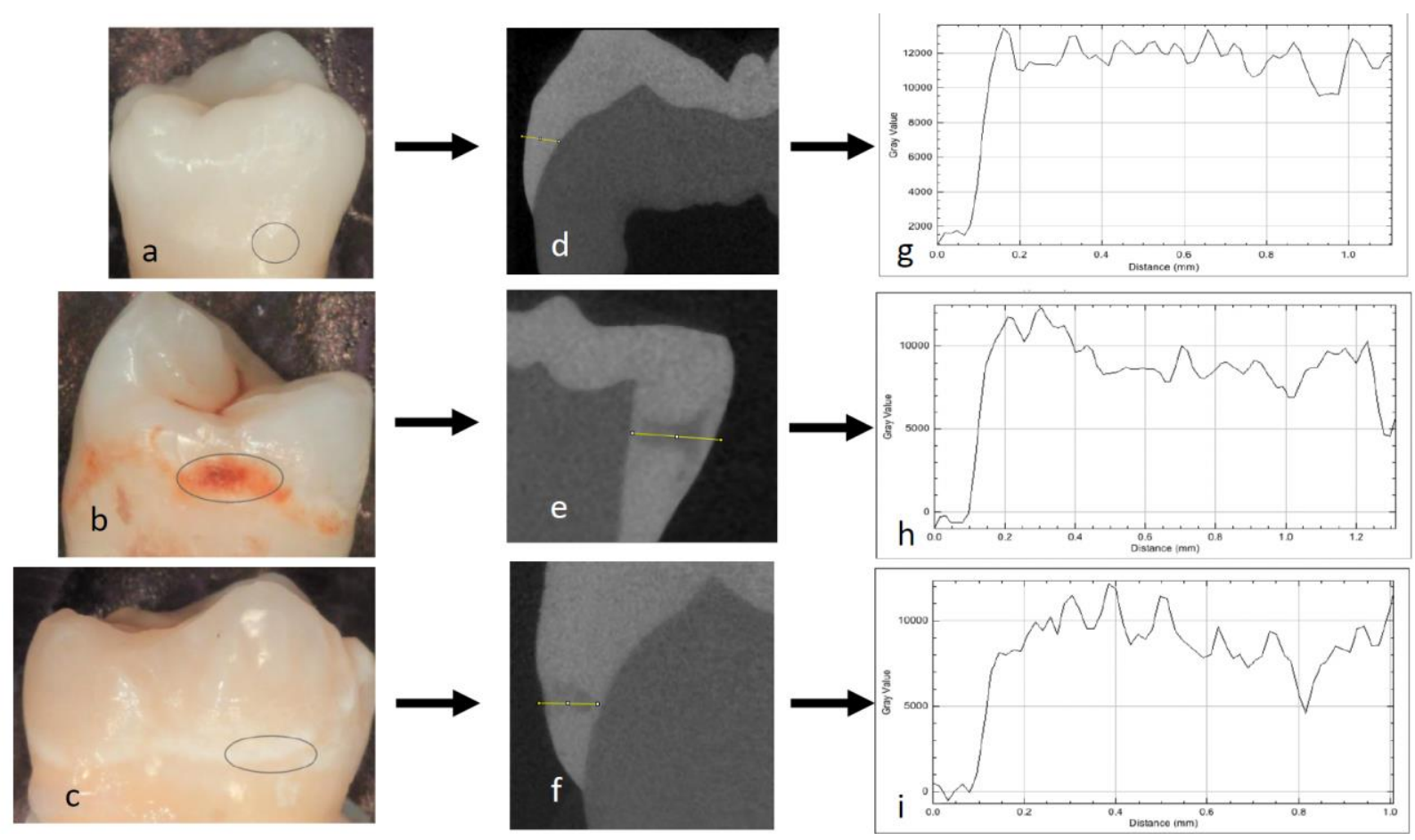

Figure 1. Clinical photographs, micro-CT slice, and plot profile for a unanimously scored ICCMS sound tooth, inactive carious lesion, and active carious lesion. Clinical photographs with the circled region of interest for a unanimously scored ICCMS sound tooth (a), inactive lesion (b), and active lesion (c). Sagittal slice captured by micro-CT of the deepest portion of the lesion with the yellow Straight Line tool used to draw a profile from the enamel surface inwards corresponding to 
ICCMS sound tooth (d), inactive lesion (e), and active lesion (f). The corresponding plot profile for each lesion is shown (g-i).

\section{Micro-CT Analysis}

ImageJ (version 1.52q, National Institutes of Health, USA) was used for image analysis [20]. Each tooth was examined as an image sequence in sagittal cross-section, using the Orthogonal Views function to confirm the region of interest and the optimal view perpendicular to the surface

of interest. Stacks were scrolled through to identify the slice corresponding to the deepest part of the lesion, which was used for analysis. The Straight Line tool was used to draw a line of interest through the middle of the lesion originating from the enamel surface and extending to the enamel-dentin junction (Figure 1d-f). A Plot Profile (Figure 1g-i) was then used to analyze the gray values along the straight line. Gray value data sets were exported for analysis.

\section{Plot Profile Analysis}

Microsoft Excel was used to run data analysis. Gray values were converted to mineral density values measured in $\mathrm{gHA} / \mathrm{cm}^{3}$ based on machine parameters. Plot Profiles from each of the measured surfaces were standardized to each other on the $\mathrm{x}$-axis by aligning the steepest portion of curves. The steepest portion of the curve represents the transition between air and enamel surface caused by an inherent micro-CT artifact, partial volume effect. The aligned curves were compiled and median mineral densities were calculated for sound, inactive and active lesions, and plotted against the distance from the surface of the lesion (Figure 2). Area under the curve (AUC) analysis was conducted for the first $96 \mu \mathrm{m}$ of the Plot Profile of each scored surface. Finally, maximum mineral density was identified within the first $144 \mu \mathrm{m}$ of the Plot Profile for each tooth. 


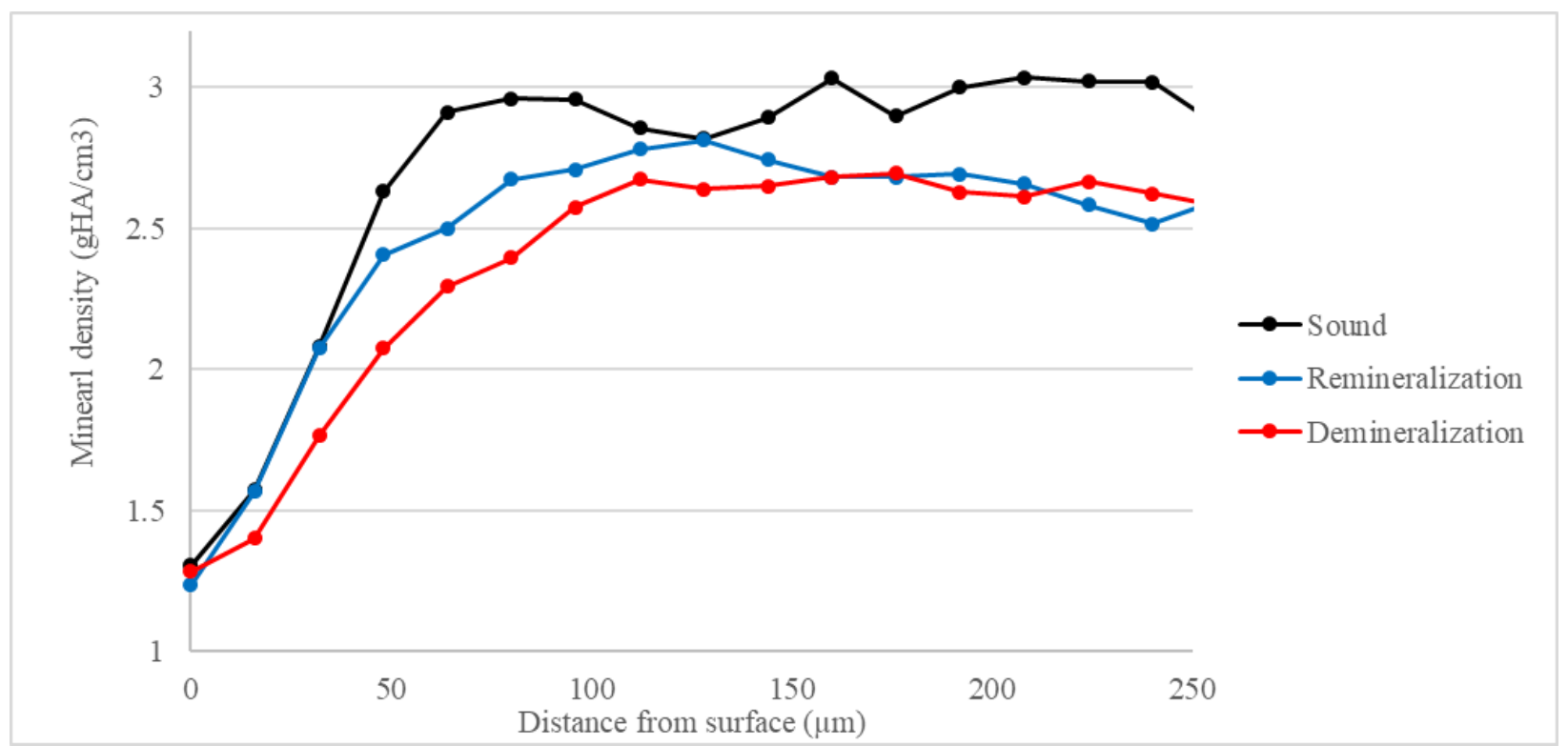

Figure 2. Median mineral density plotted against distance from enamel surface. Median mineral density of compiled sound $(n=5)$, remineralized $(n=7)$, and demineralized $(n=4)$ enamel surfaces separated according to consensus ICCMS examinations of sound, inactive lesions, or active lesions measured in the most superficial $250 \mu \mathrm{m}$ of the enamel thickness.

\section{Statistical Analysis}

Comparison between median mineral densities from micro-CT and activity scores (ICCMS) was analyzed by Kruskal-Wallis test. Agreement between inter- and intra-examiner was analyzed by Fleiss kappa coefficients. Kruskal-Wallis and Kappa tests were performed using SAS (SAS Institute, Cary, NC).

\section{Results}

Teeth demonstrating unanimous agreement among both examiners were selected to preliminarily assess differences in surface layer mineralization based on caries activity 
classification. This group $(n=16)$ consisted of smooth surfaces that were classified as sound $(n=5)$, with inactive lesions $(n=7)$, or active lesions $(n=4)$. Figure 2 illustrates the plot profile of the compiled median mineral density values for sound surfaces, remineralized and demineralized areas, demonstrating that areas clinically classified as active corresponded to a decreased (demineralized) density profile at the enamel surface layer compared to areas classified as consensus inactive and sound surfaces. Significant differences in mineral densities were observed in the mean AUC value comparison between consensus sound, active and inactive lesions from the Plot Profile Analysis, as demonstrated in Table 1.

\begin{tabular}{|c|c|c|c|c|c|c|}
\hline & \multicolumn{6}{|c|}{ Mineral density (gHA/cm3) } \\
\hline & \multicolumn{3}{|c|}{ AUC: $96 \mu \mathrm{m}$} & \multicolumn{3}{|c|}{ AUC: $144 \mu \mathrm{m}$} \\
\hline & \multicolumn{3}{|c|}{ ICCMS Exam } & \multicolumn{3}{|c|}{ ICCMS Exam } \\
\hline & Sound & Inactive & Active & Sound & Inactive & Active \\
\hline & 219.72 & 214.18 & 198.60 & 344.70 & 349.49 & 329.12 \\
\hline & 266.03 & 192.51 & 203.66 & 402.24 & 323.56 & 327.55 \\
\hline & 264.33 & 201.19 & 184.87 & 408.23 & 341.85 & 301.24 \\
\hline & 243.32 & 188.61 & 183.00 & 394.46 & 320.39 & 312.66 \\
\hline & 236.80 & 232.32 & & 382.22 & 352.50 & \\
\hline & & 214.35 & & & 342.76 & \\
\hline & & 232.49 & & & 355.53 & \\
\hline MEAN & 246.04 & 210.81 & 192.53 & 386.37 & 340.87 & 317.64 \\
\hline STD. DEV & 19.49 & 17.80 & 10.17 & 25.24 & 14.79 & 13.21 \\
\hline
\end{tabular}

Table 1. Listed AUC values from the mineral density $\left(\mathrm{gHA} / \mathrm{cm}^{3}\right)$ plot profiles of the first $96 \mu \mathrm{m}$ and $144 \mu \mathrm{m}$ of enamel for unanimous agreement teeth $(\mathrm{n}=16)$, differentiated according to gold standard ICCMS classification, along with mean and standard deviation.

Intra-examiner ICCMS activity agreement for the entire sample set $(\mathrm{n}=59)$ ranged from 0.72-0.75 kappa coefficient $(k)$, while inter-examiner agreement was $k=0.64$, demonstrating substantial agreement for each. Discriminate analysis revealed optimal AUC thresholds (taken at $96 \mu \mathrm{m}$ enamel depth) for differentiating lesions based on their ICCMS activity (Table 2). These thresholds for sound, remineralization, and demineralization were compared to ICCMS sound, 
inactive, and active scores in Table 3, with percent agreement and simple kappa calculations shown in Table 4.

\begin{tabular}{l|c}
$\begin{array}{l}\text { Enamel } \\
\text { surface } \boldsymbol{\mu C T} \\
\text { status }\end{array}$ & $\begin{array}{c}\text { AUC Threshold } \\
\text { at } 96 \boldsymbol{\mu m}\end{array}$ \\
\hline \multicolumn{1}{|c|}{ Sound } & $\geq 216$ \\
\hline Remineralized & $203 \leq$ AUC $<216$ \\
\hline Demineralized & $<203$ \\
\hline
\end{tabular}

Table 2. AUC thresholds for determining lesion demineralization at an enamel depth of $96 \mu \mathrm{m}$.

\begin{tabular}{ll|c|c|c|r|}
\multicolumn{1}{c}{} & & \multicolumn{3}{c}{ ICCMS Exam } & \\
\cline { 3 - 6 } Mineral & Detive & Inactive & Sound & Total \\
Density & Remineralized & 11 & 6 & 4 & 21 \\
Classification & Sound & 0 & 5 & 12 & 21 \\
\cline { 2 - 6 } & Total & 15 & 21 & 23 & 59
\end{tabular}

Table 3. Comparison of ICCMS gold standard examiner against the mineral density classification using the established plot profile AUC thresholds for sound, remineralized, and demineralized surfaces.

\begin{tabular}{r|cc} 
& \% Agreement & Simple Kappa \\
\hline Unanimous agreement $(n=16)$ & 62.5 & 0.46 \\
Disagreement $(n=43)$ & 53.5 & 0.31 \\
\hline All surfaces $(n=59)$ & 55.9 & 0.34
\end{tabular}

Table 4. Percent agreement and kappa values of the differentiation between ICCMS sound, inactive, and active lesions for the near-unanimous agreement and disagreement teeth sets using AUC thresholds for the first $96 \mu \mathrm{m}$.

Further analysis was conducted to assess how the ICCMS classification of active against non-active (including sound and inactive lesions) performed against the micro-CT thresholds for the demineralization group to the "non-demineralization" group (Table 5). Lastly, the demineralization group was compared to the remineralization group as a correlate of ICCMS active against inactive detection (Table 6). Comparisons of percent agreement, kappa values, specificity and sensitivity are shown in Table 7. The percent agreement and kappa values for the 
differentiation of caries activity using micro-CT demineralization vs. remineralization using maximum mineral density value in the first $144 \mu \mathrm{m}$ of enamel were $59.3 \%$ and 0.39 , respectively.

\begin{tabular}{ll|c|c|r|} 
& & \multicolumn{3}{c}{ ICCMS Exam } \\
\cline { 2 - 4 } Mineral & Active & Inactive/Sound & Total \\
Density & Non- & 4 & 10 & 21 \\
Classification & Demineralized & & 34 & 38 \\
\cline { 2 - 4 } & Total & 15 & 44 & 59
\end{tabular}

Table 5. Comparison of ICCMS gold standard examiner against the mineral density classification using the established plot profile AUC thresholds for demineralized and "non-demineralized" lesions.

\begin{tabular}{llcc|r|} 
& & \multicolumn{3}{c}{ ICCMS Exam } \\
\cline { 2 - 4 } & & Active & Inactive & Total \\
\cline { 1 - 4 } $\begin{array}{l}\text { Mineral } \\
\text { Density } \\
\text { Classification }\end{array}$ & Demineralized & 11 & 6 & 17 \\
\cline { 2 - 4 } & Remineralized & 4 & 10 & 14 \\
\hline & Total & 15 & 16 & 31
\end{tabular}

Table 6. Comparison of ICCMS gold standard examiner against the mineral density classification using the established plot profile AUC thresholds for demineralized and remineralized surfaces.

\begin{tabular}{|c|c|c|c|c|c|c|}
\hline & $\begin{array}{c}\% \\
\text { Agreement }\end{array}$ & $\begin{array}{l}\text { Simple } \\
\text { Kappa }\end{array}$ & Sensitivity & Specificity & $\begin{array}{l}\text { False } \\
\text { Pos. }\end{array}$ & $\begin{array}{l}\text { False } \\
\text { Neg. }\end{array}$ \\
\hline $\begin{array}{r}\text { Active vs. Inactive vs. } \\
\text { Sound }\end{array}$ & 55.9 & 0.34 & -- & -- & -- & -- \\
\hline Active vs. Inactive/Sound & 76.3 & 0.45 & 0.73 & 0.77 & 0.48 & 0.11 \\
\hline Active vs. Inactive only & 67.7 & 0.36 & 0.73 & 0.63 & 0.35 & 0.29 \\
\hline
\end{tabular}

Table 7. Percent agreement, kappa values, sensitivity, specificity, false positives, and false negatives of the differentiation between micro-CT demineralization vs. non-demineralization using AUC for the first $96 \mu \mathrm{m}$. 


\section{Discussion}

This study shows statistically significant differences in mineral density of the enamel surface layer corresponding to areas classified via ICCMS examination as initial active and inactive carious lesions and sound smooth surfaces. Areas classified as active by the visual-tactile method revealed lower mineral content in the outermost $96 \mu \mathrm{m}$ of enamel when compared to areas classified as inactive or sound. This characteristic was then used to differentiate the status of the enamel lesions via mineral density thresholds.

Even for our experienced, calibrated gold standard examiners, ICCMS assessment of caries activity for early carious lesions remained a significant challenge. The substantial agreement for intra-kappa (0.72-0.75) and inter-kappa (0.64) activity assessment for this sample population is consistent with literature values for activity assessment of early carious lesions [1,21-23]. Therefore, the need remains to establish a non-invasive gold standard for in vitro studies in order to validate potential caries activity diagnostic methods.

It has been shown that dentin mineral density is significantly lower in active compared to inactive lesions [13]; however, assessing average mineral density values in enamel, especially in non-cavitated lesions, is a challenge since it requires manually tracing an area of interest that has poorly defined boundaries, as initial lesions characteristically do [14]. An alternative methodological approach using micro-CT to assess lesion activity has been to follow a mineral density plot profile of the lesion from the enamel surface towards the pulp $[14,15,24]$. The line plot method minimizes the subjectivity of defining borders around a lesion and provides a visual depiction of the remineralization that occurs with caries arrestment while following the histopathology of lesion progression [25]. Previous work identified that brown spot lesions, commonly recognized as a sign of arrestment, have a thicker surface layer of increased mineral 
density compared to white spot lesions [26]. Other work has identified in vivo remineralization of the surface layer of smooth surface lesions occurring after arresting caries from white spot lesions [27]. These findings point to a remineralization occurring from the outer surface of the enamel inwards once a lesion becomes arrested.

Our findings demonstrated identifiable differences in mineral density between enamel surfaces classified via ICCMS examination as sound surfaces, inactive lesions, and active lesions. Qualitative analysis from the median Plot Profile curves (Fig. 2) illustrates a difference in mineral density values between ICCMS sound, inactive, and active lesions in the most superficial $\sim 200$ $\mu \mathrm{m}$ of enamel. Areas classified as ICCMS sound surfaces and inactive lesions tended to have observably higher mineral densities in the surface layer compared to the areas classified as active lesions (Table 1).

Furthermore, our findings agree with previous work that the remineralization process in inactive carious lesions occurs in a characteristic pattern from the external surface of the lesion inwards [15]. Inactivation, or arrestment, of carious lesions resulted in a progressive increase in mineral content compared to active lesions, beginning with a characteristic increase in mineral density in the outermost surface of enamel (Fig.1h, Fig. 2).

Further quantitative analysis of the AUC plot profiles identified a potential method for classifying lesions as sound, inactive, or active using different thresholds for mineral density within the first $96 \mu \mathrm{m}$ (Table 2). The $96 \mu \mathrm{m}$ threshold for calculating AUC was optimal because it showed the greatest variation in the surface layer amongst the sound, inactive, and active lesions. Supplementary Fig. S1 illustrates this optimal $96 \mu \mathrm{m}$ threshold when assessing the composite of all 59 aligned curves. By using the AUC of the plot profiles, micro-CT artifacts such as random noise and partial volume effect are minimized by sampling the entire outer enamel surface layer. 
Although beam hardening effect and surface alignment remain a challenge with micro-CT quantitative comparison, careful alignment was completed via the steepest portion of the curve, correlating to the transition between air and enamel.

Within our sample size, the ICCMS examination when classifying sound, inactive lesions, and active lesions demonstrates fair strength of agreement with the AUC method for classifying lesions (Table 4). However, as seen in Table 3, a significant portion of the percent disagreement came from the overlapping mineral content of remineralized lesions and sound surfaces, a finding that makes sense as an inactive lesion that has remineralized for a number of years may return to a mineral density consistent with sound surfaces. Therefore, our further analysis investigated the ability of micro-CT to identify ICCMS active lesions from the entire sample set (Table 5). As seen in Table 7, the ability of micro-CT to identify active lesions via AUC demineralization showed moderate agreement with our gold standard examiner, with good sensitivity and specificity values. The greatest clinical benefit to utilizing the micro-CT AUC plot profiles as an indicator of caries activity is the ability to differentiate active initial carious lesions from inactive initial carious lesions, which remains one of the greatest clinical challenges in caries diagnosis. Therefore, a final analysis was conducted comparing the results of the micro-CT demineralization against remineralization classification, correlating to the clinical judgement between active and inactive initial carious lesions (Table 6-7). The fair agreement between the micro-CT classification and our gold standard examiner points to the continued challenge of caries activity diagnosis even for a calibrated, experienced gold standard examiner under ideal conditions.

We acknowledge the limitation of this study utilizing ex vivo teeth to classify caries activity when the only current gold standard in caries activity diagnosis is lesion progression. However, the preservation and analysis of ex vivo samples allowed for the most accurate snapshot of caries 
activity at the time the teeth were extracted. In contrast to studies with artificially simulated caries on enamel samples or studies using ex vivo samples from different species, this study investigated the ability of examiners to detect caries activity in the most similar manner to the clinical activity diagnosis.

To our knowledge this is the first study to utilize a substantial sample size-in vivo, ex vivo, or otherwise - to propose quantifiable differences in the surface layer between sound surfaces and inactive and active lesions. As has been suggested by previous studies, remineralization during the inactivation of carious lesions tends to create a hard surface layer on the outermost part of enamel $[26,28]$. This study provides increased evidence for the surface layer hypothesis, as we identified an increase in mineral density of the surface layer for inactive lesions compared to active demineralization sites. Even further, this study utilizes this working hypothesis to identify a quantifiable reference standard that can be used for future caries activity validation studies. The thresholds established using the samples in this study were then applied to the ICCMS gold standard examiner to reveal the challenge that remains in caries activity classification.

Future work should continue to optimize the thresholds established by this study, as well as investigate the optimal surface layer depth for AUC measurements, building on the principles of this study to further validate the AUC line plot method for using micro-CT as a gold standard for caries activity diagnosis.

\section{References}

1. Ismail, A. I. et al. The International Caries Detection and Assessment System (ICDAS): an integrated system for measuring dental caries. Community Dent Oral Epidemiol 35, 170-178 (2007).

2. Nyvad, B., Machiulskiene, V. \& Baelum, V. Reliability of a new caries diagnostic system differentiating between active and inactive caries lesions. Caries Res. 33, 252-260 (1999). 
3. Ekstrand, K. R., Martignon, S., Ricketts, D. J. N. \& Qvist, V. Detection and activity assessment of primary coronal caries lesions: a methodologic study. Oper Dent 32, 225-235 (2007).

4. Tikhonova, S. M., Feine, J. S., Pustavoitava, N. N. \& Allison, P. J. Reproducibility and Diagnostic Outcomes of Two Visual-Tactile Criteria Used by Dentists to Assess Caries Lesion Activity: A Cross-Over Study. CRE 48, 126-136 (2014).

5. Slayton, R. L. et al. Evidence-based clinical practice guideline on nonrestorative treatments for carious lesions: A report from the American Dental Association. The Journal of the American Dental Association 149, 837-849.e19 (2018).

6. MacGregor, A. B. The position and extent of acid in the carious process. Archives of Oral Biology 4, 86-91 (1961).

7. Ekstrand, K. R., Ricketts, D. N. J., Kidd, E. A. M., Qvist, V. \& Schou, S. Detection, diagnosing, monitoring and logical treatment of occlusal caries in relation to lesion activity and severity: An in vivo examination with histological validation. Caries Research; Basel 32, 247-54 (1998).

8. Drancourt, N. et al. Carious lesion activity assessment in clinical practice: a systematic review. Clin Oral Investig (2019). doi:10.1007/s00784-019-02839-7

9. Huysmans, M.-C. D. N. J. M. \& Longbottom, C. The challenges of validating diagnostic methods and selecting appropriate gold standards. J. Dent. Res. 83 Spec No C, C48-52 (2004).

10. Soviero, V. M., Leal, S. C., Silva, R. C. \& Azevedo, R. B. Validity of MicroCT for in vitro detection of proximal carious lesions in primary molars. J Dent 40, 35-40 (2012).

11. Bjørndal, L., Carlsen, O., Thuesen, G., Darvann, T. \& Kreiborg, S. External and internal macromorphology in 3D-reconstructed maxillary molars using computerized X-ray microtomography. International Endodontic Journal 32, 3-9 (1999).

12. Dowker, S. E. P., Davis, G. R., Elliott, J. C. \& Wong, F. S. L. X-ray microtomography: 3dimensional imaging of teeth for computer-assisted learning. European Journal of Dental Education 1, 61-65 (1997).

13. Neves, A. A., Vargas, D. O. A., Santos, T. M. P., Lopes, R. T. \& Sousa, F. B. Is the morphology and activity of the occlusal carious lesion related to the lesion progression stage? Arch. Oral Biol. 72, 33-38 (2016).

14. Huang, T. T. Y., Jones, A. S., He, L. H., Darendeliler, M. A. \& Swain, M. V. Characterisation of enamel white spot lesions using X-ray micro-tomography. Journal of Dentistry 35, 737-743 (2007).

15. Nakata, K., Nikaido, T., Nakashima, S., Nango, N. \& Tagami, J. An approach to normalizing micro-CT depth profiles of mineral density for monitoring enamel remineralization progress. Dent Mater J 31, 533-540 (2012).

16. Cochrane, N. J. et al. An X-ray Microtomographic Study of Natural White-spot Enamel Lesions. J Dent Res 91, 185-191 (2012). 
17. Groeneveld, A. \& Arends, J. Influence of $\mathrm{pH}$ and Demineralization Time on Mineral Content, Thickness of Surface Layer and Depth of Artificial Caries Lesions. CRE 9, 36-44 (1975).

18. Arends, J. \& Christoffersen, J. Invited Review Article: The Nature of Early Caries Lesions in Enamel. J Dent Res 65, 2-11 (1986).

19. International Caries Detection and Assessment System (ICDAS) Coordinating Committee. Rationale and Evidence for the International Caries Detection and Assessment System (ICDAS II). (2012). at <http://www.icdas.org>

20. Rueden, C. T. et al. ImageJ2: ImageJ for the next generation of scientific image data. $B M C$ Bioinformatics 18, (2017).

21. Carvalho, J. C., Qvist, V., Aimée, N. R., Mestrinho, H. D. \& Bakhshandeh, A. Diagnosis, Risk Assessment, and Treatment Decisions for Occlusal Caries: A Survey from the Danish Public Dental Health Service. CRE 52, 58-70 (2018).

22. Ekstrand, K. R., Ricketts, D. N. J., Longbottom, C. \& Pitts, N. B. Visual and Tactile Assessment of Arrested Initial Enamel Carious Lesions: An in vivo Pilot Study. CRE 39, 173-177 (2005).

23. Ando, M., Eckert, G. J. \& Zero, D. T. Preliminary Study to Establish a Relationship between Tactile Sensation and Surface Roughness. CRE 44, 24-28 (2010).

24. Mei, M. L. et al. An ex vivo study of arrested primary teeth caries with silver diamine fluoride therapy. J Dent 42, 395-402 (2014).

25. Bjørndal, L. \& Thylstrup, A. A structural analysis of approximal enamel caries lesions and subjacent dentin reactions. Eur. J. Oral Sci. 103, 25-31 (1995).

26. Shahmoradi, M. \& Swain, M. V. Micro-CT analysis of naturally arrested brown spot enamel lesions. Journal of Dentistry 56, 105-111 (2017).

27. Rosa, T.C. et al. The bacterial microbiome and metabolome in caries progression and arrest. Journal of Oral Microbiology 13, 1-15 (2021).

28. Ando, M. et al. Objective and quantitative assessment of caries lesion activity. Journal of Dentistry 78, 76-82 (2018).

\section{Acknowledgements}

Biomedical Research Imaging Center (UNC School of Medicine) conducted the micro-CT imaging. This work was supported by the University of North Carolina Adams School of Dentistry and Dental Foundation of North Carolina. 


\section{Author Contributions}

AMH contributed to acquisition of the data for the work, drafting the manuscript. AAN contributed to data analysis, review of the manuscript. KM contributed to data analysis, review of the manuscript. ABV contributed to acquisition of the data for the work, review of the manuscript. AFZ contributed to acquisition of the data for the work, review of the manuscript. AAR contributed to design of the work, acquisition of data for the work, interpretation of the data, review of the manuscript.

\section{Additional Information}

The authors declare no competing interests.

The authors state that all methods were carried out in accordance with relevant guidelines and regulations.

The authors state that the study was reviewed by the Office of Human Research Ethics, Study \#:15-1945, which has determined that this submission does not constitute human subjects research as defined under federal regulations [45 CFR 46.102 (d or f) and 21 CFR 56.102(c)(e)(l)], so the study was classified as NHSR, since teeth included in this study were considered discarded material and no one on the study team has access to the subject identifiers linked to the specimens. 


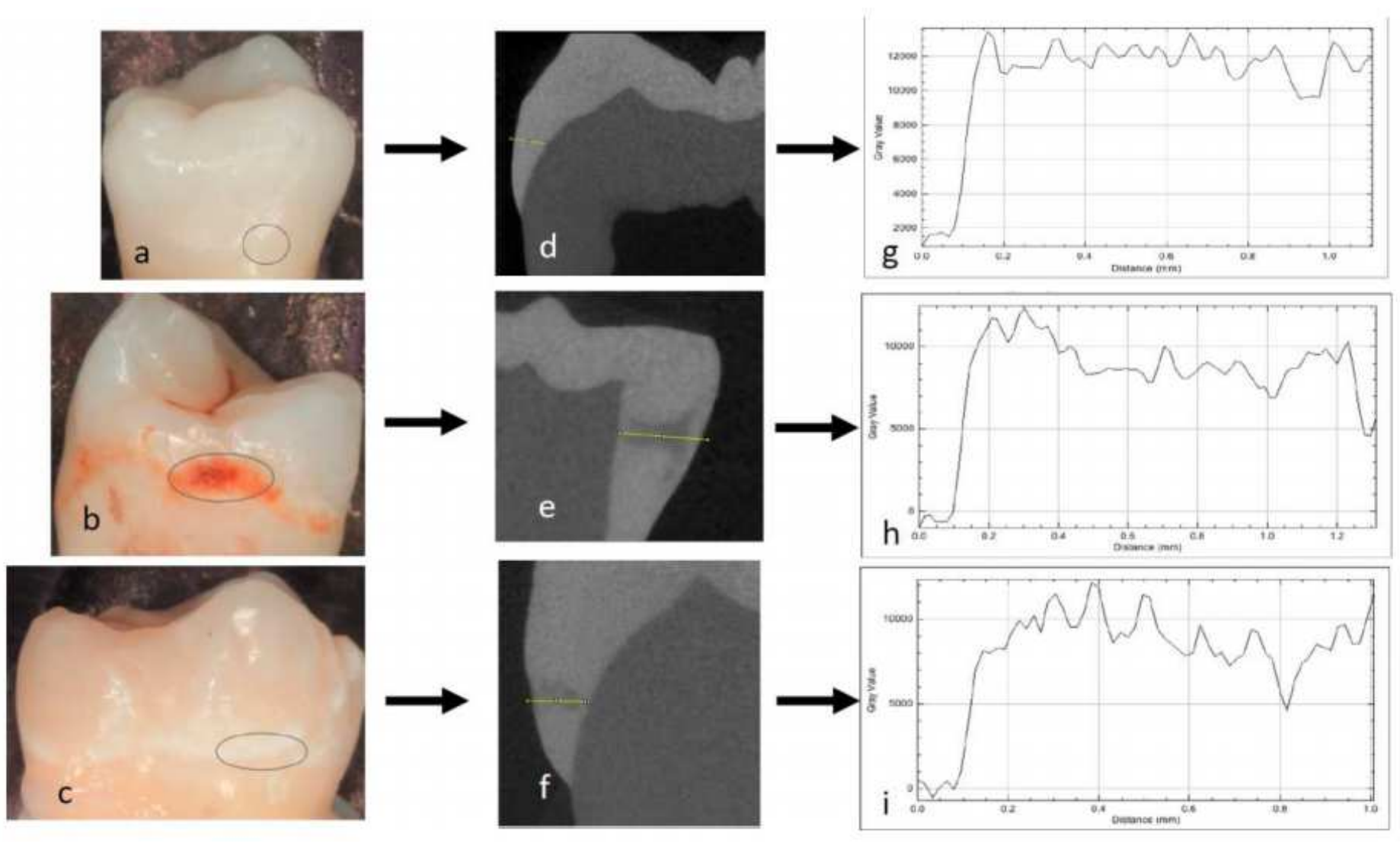

\section{Figure 1}

Clinical photographs, micro-CT slice, and plot profile for a unanimously scored ICCMS sound tooth, inactive carious lesion, and active carious lesion. Clinical photographs with the circled region of interest for a unanimously scored ICCMS sound tooth (a), inactive lesion (b), and active lesion (c). Sagittal slice captured by micro-CT of the deepest portion of the lesion with the yellow Straight Line tool used to draw a profile from the enamel surface inwards corresponding to ICCMS sound tooth (d), inactive lesion (e), and active lesion (f). The corresponding plot profile for each lesion is shown ( $g-i)$. 


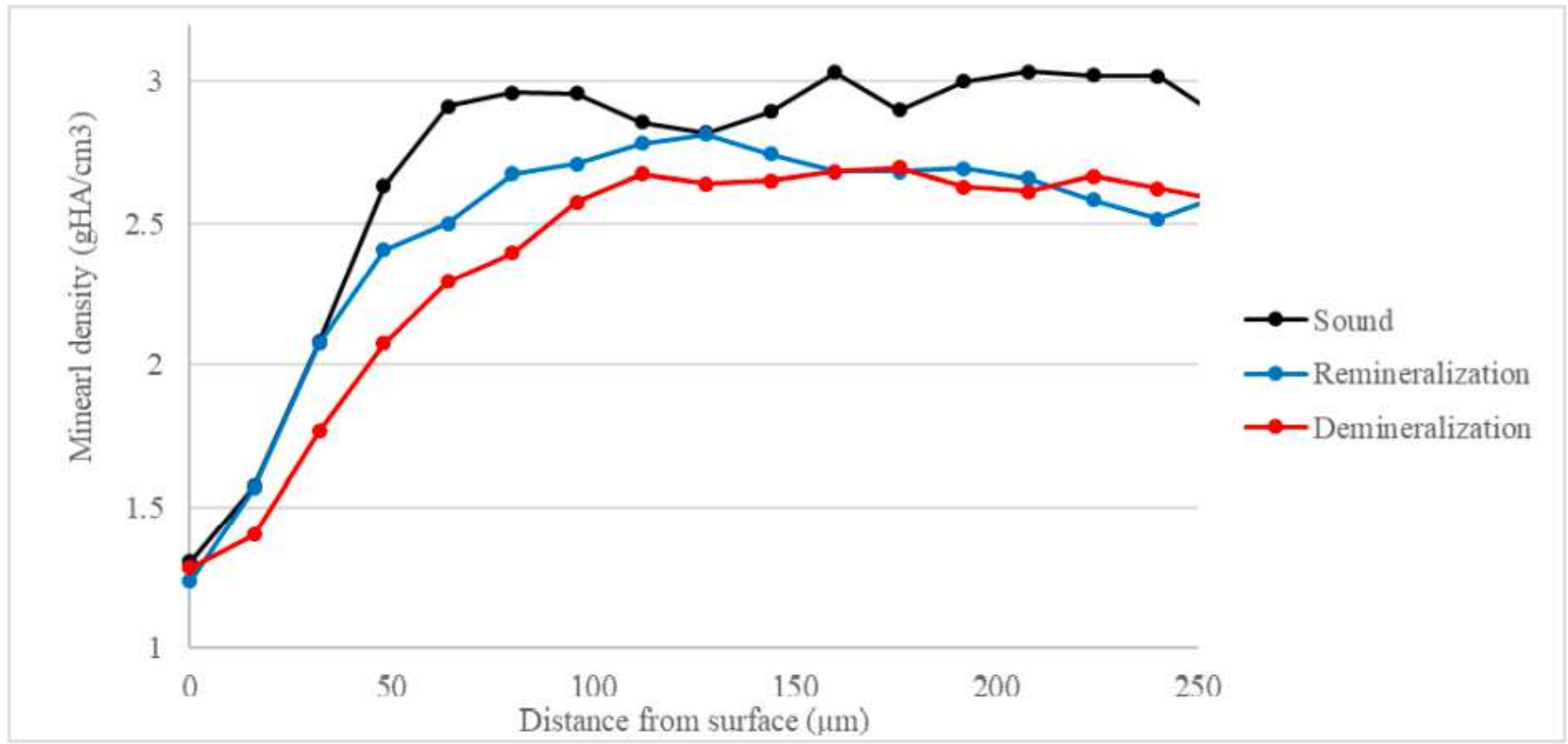

Figure 2

Median mineral density plotted against distance from enamel surface. Median mineral density of compiled sound $(n=5)$, remineralized $(n=7)$, and demineralized $(n=4)$ enamel surfaces separated according to consensus ICCMS examinations of sound, inactive lesions, or active lesions measured in the most superficial $250 \mu \mathrm{m}$ of the enamel thickness.

\section{Supplementary Files}

This is a list of supplementary files associated with this preprint. Click to download.

- SupplementaryFigureS1.pdf 